\title{
SYNTHETIC DIFFERENTIAL GEOMETRY OF CHEN'S ITERATED INTEGRALS
}

\author{
HIROKAZU NISHIMURA
}

\begin{abstract}
Chen's iterated integrals are treated within synthetic differential geometry. The main result is that iterated integrals produce a subcomplex of the de Rham complex on the free path space as well as based path spaces.
\end{abstract}

\section{INTRODUCTION}

Chen's iterated integrals have been introduced and investigated in [1-3] and others. As far as we know, there is only one volume on them, namely, [9], though it is unfortunately written not in English but in Japanese. Chen has established two fundamental theorems on them, the first claiming that the cohomology of the bar complex $\mathcal{B}^{*}(M)$ generated by Chen's iterated integrals on the loop space $\Omega M$ of a smooth manifold $M$ is no other than the cohomology of the loop space $\Omega M$, so long as $M$ is simply connected, while the second assertion is that

$$
\mathcal{F}^{-k} H^{0}\left(\mathcal{B}^{*}(M)\right) \cong \operatorname{Hom}\left(\mathbf{Z} \pi_{1}\left(M, x_{0}\right) / J^{k+1}, \mathbf{R}\right)
$$

where $\mathcal{F}^{-k}$ is the filtration determined by Chen's iterated integrals of length $k$ or less, $\mathbf{Z} \pi_{1}\left(M, x_{0}\right)$ denotes the group ring of the fundamental group $\pi_{1}\left(M, x_{0}\right)$, and $J$ stands for the kernel of the augmentation mapping $\mathbf{Z} \pi_{1}\left(M, x_{0}\right) \rightarrow \mathbf{Z}$. Chen's iterated integrals have been applied successfully to various branches of mathematics such as to Vassiliev invariants of knots and braids ([8] and [10]), algebraic cycles ([5]) and multiple zeta functions ( [4] and [16]).

The principal objective in this paper is to establish that Chen's iterated integrals yield a subcomplex of the de Rham complex on the free path space $\mathcal{P} M$ as well as the path space $\mathcal{P}_{x_{1}} M$ with the starting point based upon $x_{1} \in M$, the path space $\mathcal{P}^{x_{2}} M$ with the terminating point based upon $x_{2} \in M$ and the path space $\mathcal{P}_{x_{1}}^{x_{2}} M$ with both the starting and terminating points based upon $x_{1} \in M$ and $x_{2} \in M$ respectively within our favorite framework of synthetic differential geometry ([7] and [11]). Chen's two fundamental theorems will be dealt with synthetically in subsequent papers.

Even if $M$ is a finite-dimensional smooth manifold, the free path space $\mathcal{P} M$ as well as the path space $\mathcal{P}_{x_{1}} M$ with the starting point based upon $x_{1}$, the path space $\mathcal{P}^{x_{2}} M$ with the terminating point based upon $x_{2}$ and the path space $\mathcal{P}_{x_{1}}^{x_{2}} M$ with both the starting and terminating points based upon $x_{1}$ and $x_{2}$ respectively

$M S C$ (2010): primary 58A10, 55D35, 51K10.

Keywords: synthetic differential geometry, path space, Chen's iterated integrals, Hochschild complex, bar complex. 
is generally infinite-dimensional in essence, so that we must choose an adequate framework for infinite-dimensional differential geometry in order to deal with these spaces. Chen has chosen so-called diffeology, in which plots enable one to do constructions and computations with coordinates. For an excellent volume on diffeology, one is referred to [6]. Since we have chosen synthetic differential geometry and we assume $M$ only to be a microlinear space, we are coerced into doing everything in a coordinate-free way.

In synthetic differential geometry, one has to do everything within an esoteric topos, which alienates many mathematicians. However we could emancipate synthetic differential geometry from topos theory, resulting in axiomatic differential geometry, in which Weil functors play a pivotal role. For the first steps in axiomatic differential geometry, one is referred to $[12,13]$ and [14]. For an excellent investigation on the relationship among a few infinite-dimensional differential geometries (including diffeology) from the standpoint of category theory, one is referred to [15].

\section{Preliminaries}

The reader is referred to [7] and [11] for synthetic differential geometry. In particular, the reader is assumed to be familiar with the first four chapters of [11].

Notation 2.1. We denote by $M$ an arbitrary microlinear space.

Notation 2.2. We denote by $I$ the unit interval $[0,1]$.

Remark 2.3. As is discussed in $\S 3.2$ of [11], vector fields on $M$ can be viewed from three related but distinct standpoints. The first is to see them orthodoxically as mappings $M \rightarrow M^{D}$ (sections of tangent bundles), the second is to put them down as mappings $D \times M \rightarrow M$ (infinitesimal flow), and the third is, most radically, to regard them as mappings $D \rightarrow M^{M}$ (infinitesimal transformation), though we prefer the third viewpoint most.

Notation 2.4. We denote by $\mathcal{A}^{p}(M)$ the totality of differential forms on $M$ of degree $p, \mathcal{A}(M)$ designating the totality of differential forms on $M$.

Notation 2.5. We denote by $\mathbf{d}$ the exterior differentiation. Given a vector field $X$ on $M$, we denote by $\mathbf{i}_{X}$ and $\mathbf{L}_{X}$ the interior product and the Lie derivative with respect to the vector field $X$ respectively.

Remark 2.6. We have a natural pairing

$$
\left\langle\left(\gamma ; d_{1}, \ldots, d_{p}\right), \omega\right\rangle=d_{1} \ldots d_{p} \omega(\gamma)
$$

for any $\left(\gamma ; d_{1}, \ldots, d_{p}\right) \in M^{D^{p}} \times D^{p}$ and any $\omega \in \mathcal{A}^{p}(M)$. Indeed, differential forms can be characterized as mappings on $M^{D^{p}} \times D^{p}$ having certain properties, for which the reader is referred to Proposition 2 in $\S 4.2$ of [11].

The following is one of the three Cartan formulas for differential forms and will be used in our discussions. 
Theorem 2.7. Given a vector field $X$ on $M$, we have

$$
\mathbf{L}_{X}=\mathbf{d i} \mathbf{i}_{X}+\mathbf{d i} \mathbf{i}_{X}
$$

\section{Simple Integrals}

Notation 3.1. We denote by $\mathcal{P} M$ the set

$$
\mathcal{P} M=M^{I} .
$$

Notation 3.2. We denote by $\varphi$ the mapping $\varphi: I \times \mathcal{P} M \rightarrow M$ defined by

$$
\varphi(t, \theta)=\theta(t)
$$

for any $(t, \theta) \in I \times \mathcal{P} M$.

Notation 3.3. Given $t \in I$, we denote by $\iota_{t}: \mathcal{P} M \rightarrow I \times \mathcal{P} M$ the mapping

$$
\theta \in \mathcal{P} M \mapsto(t, \theta) \in I \times \mathcal{P} M .
$$

Notation 3.4. Given $t \in I$, we denote by $\varphi_{t}$ the mapping

$$
\varphi \circ \iota_{t}: \mathcal{P} M \rightarrow M \text {. }
$$

Notation 3.5. We denote by $\frac{\partial}{\partial t}$ the vector field

$$
(d,(t, \theta)) \in D \times(I \times \mathcal{P} M) \mapsto(t+d, \theta) \in I \times \mathcal{P} M
$$

on $I \times \mathcal{P} M$.

Notation 3.6. Given $\omega \in \mathcal{A}^{p}(M)$ with $p$ being a positive integer and $t \in I$, we write

$$
(\omega)_{t}^{*} \in \mathcal{A}^{p-1}(\mathcal{P} M)
$$

for

$$
\iota_{t}^{*} \mathbf{i} \frac{\partial}{\partial t} \varphi^{*} \omega
$$

Proposition 3.7. Given $\omega_{1} \in \mathcal{A}^{p_{1}}(M)$ and $\omega_{2} \in \mathcal{A}^{p_{2}}(M)$ with $p_{1}$ and $p_{2}$ being positive integers, we have

$$
\left(\omega_{1} \wedge \omega_{2}\right)_{t}^{*}=\left(\omega_{1}\right)_{t}^{*} \wedge \varphi_{t}^{*} \omega_{2}+(-1)^{p_{1}} \varphi_{t}^{*} \omega_{1} \wedge\left(\omega_{2}\right)_{t}^{*}
$$

for any $t \in I$.

Proof. We have

$$
\begin{aligned}
\left(\omega_{1} \wedge \omega_{2}\right)_{t}^{*} & =\iota_{t}^{*} \mathbf{i}_{\frac{\partial}{\partial t}} \varphi^{*}\left(\omega_{1} \wedge \omega_{2}\right) \\
& =\iota_{t}^{*} \mathbf{i}_{\frac{\partial}{\partial t}}\left(\varphi^{*} \omega_{1} \wedge \varphi^{*} \omega_{2}\right) \\
& =\iota_{t}^{*}\left(\mathbf{i}_{\frac{\partial}{\partial t}} \varphi^{*} \omega_{1} \wedge \varphi^{*} \omega_{2}+(-1)^{p_{1}} \varphi^{*} \omega_{1} \wedge \mathbf{i}_{\frac{\partial}{\partial t}} \varphi^{*} \omega_{2}\right) \\
& =\iota_{t}^{*} \mathbf{i}_{\frac{\partial}{\partial t}} \varphi^{*} \omega_{1} \wedge \iota_{t}^{*} \varphi^{*} \omega_{2}+(-1)^{p_{1}} \iota_{t}^{*} \varphi^{*} \omega_{1} \wedge \iota_{t}^{*} \mathbf{i}_{\frac{\partial}{\partial t}} \varphi^{*} \omega_{2} \\
& =\left(\omega_{1}\right)_{t}^{*} \wedge \varphi_{t}^{*} \omega_{2}+(-1)^{p_{1}} \varphi_{t}^{*} \omega_{1} \wedge\left(\omega_{2}\right)_{t}^{*} .
\end{aligned}
$$


Definition 3.8. Given a mapping $\widetilde{\omega}: I \rightarrow \mathcal{A}^{p}(\mathcal{P} M)$ with $p$ being a natural number and $s, t \in I$, we define

$$
\int_{s}^{t} \widetilde{\omega}(u) \mathbf{d} u \in \mathcal{A}^{p}(\mathcal{P} M)
$$

to be such that

$$
\begin{aligned}
& \left\langle\left(\gamma ; d_{1}, \ldots, d_{p}\right), \int_{s}^{t} \widetilde{\omega}(u) \mathbf{d} u\right\rangle \\
= & \int_{s}^{t}\left\langle\left(\gamma ; d_{1}, \ldots, d_{p}\right), \widetilde{\omega}(u)\right\rangle \mathbf{d} u
\end{aligned}
$$

for any $\left(\gamma ; d_{1}, \ldots, d_{p}\right) \in(\mathcal{P} M)^{D^{p}} \times D^{p}$.

It is easy to see that

Proposition 3.9. Given a mapping $\widetilde{\omega}: I \rightarrow \mathcal{A}^{p}(\mathcal{P} M)$ with p being a natural number and $s, t \in I$, we have

$$
\mathbf{d} \int_{s}^{t} \widetilde{\omega}(u) \mathbf{d} u=\int_{s}^{t} \mathbf{d} \widetilde{\omega}(u) \mathbf{d} u .
$$

Now we are ready to give a definition of Chen's single integral, which is the starting point of his iterated integrals.

Definition 3.10. Given $\omega \in \mathcal{A}^{p}(M)$ with $p$ being a positive integer and $s, t \in I$, we define

$$
\int_{s}^{t} \omega \in \mathcal{A}^{p-1}(\mathcal{P} M)
$$

to be

$$
\int_{s}^{t}(\omega)_{u}^{*} \mathbf{d} u
$$

Notation 3.11. Given $\omega \in \mathcal{A}^{p}(M)$ with $p$ being a positive integer, we write

$$
\int \omega \in \mathcal{A}^{p-1}(\mathcal{P} M)
$$

for

$$
\int_{0}^{1} \omega
$$

Proposition 3.12. Given $\omega \in \mathcal{A}^{p}(M)$ with $p$ being a positive integer and $t \in I$, we have

$$
\mathbf{d}(\omega)_{t}^{*}=\iota_{t}^{*} \mathbf{L}_{\frac{\partial}{\partial t}} \varphi^{*} \omega-(\mathbf{d} \omega)_{t}^{*}
$$

Proof. We have

$$
\begin{aligned}
\mathbf{d}(\omega)_{t}^{*} & =\mathbf{d} \iota_{t}^{*} \mathbf{i}_{\frac{\partial}{\partial t}} \varphi^{*} \omega \\
& =\iota_{t}^{*} \mathbf{d i}_{\frac{\partial}{\partial t}} \varphi^{*} \omega \\
& =\iota_{t}^{*}\left(\mathbf{L}_{\frac{\partial}{\partial t}}-\mathbf{i}_{\frac{\partial}{\partial t}} \mathbf{d}\right) \varphi^{*} \omega
\end{aligned}
$$


[by Theorem 2.7]

$$
\begin{aligned}
& =\iota_{t}^{*} \mathbf{L}_{\frac{\partial}{\partial t}} \varphi^{*} \omega-\iota_{t}^{*} \mathbf{i}_{\frac{\partial}{\partial t}} \mathbf{d} \varphi^{*} \omega \\
& =\iota_{t}^{*} \mathbf{L}_{\frac{\partial}{\partial t}} \varphi^{*} \omega-(\mathbf{d} \omega)_{t}^{*} .
\end{aligned}
$$

Corollary 3.13. Let $t \in I$ and $d \in D$. Given $\omega \in \mathcal{A}^{p}(M)$ with $p$ being a positive integer, we have

$$
\mathbf{d} \int_{t}^{t+d} \omega=-\int_{t}^{t+d} \mathbf{d} \omega-\varphi_{t}^{*} \omega+\varphi_{t+d}^{*} \omega
$$

Proof. It suffices to show that

$$
\begin{aligned}
& \left\langle\left(\gamma ; d_{1}, \ldots, d_{p}\right), \mathbf{d} \int_{t}^{t+d} \omega\right\rangle \\
= & \left\langle\left(\gamma ; d_{1}, \ldots, d_{p}\right),-\int_{t}^{t+d} \mathbf{d} \omega-\varphi_{t}^{*} \omega+\varphi_{t+d}^{*} \omega\right\rangle
\end{aligned}
$$

for any $\left(\gamma ; d_{1}, \ldots, d_{p}\right) \in M^{D^{p}} \times D^{p}$, which follows from the following computation:

$$
\begin{aligned}
& \left\langle\left(\gamma ; d_{1}, \ldots, d_{p}\right), \mathbf{d} \int_{t}^{t+d} \omega\right\rangle \\
= & \left\langle\partial\left(\gamma ; d_{1}, \ldots, d_{p}\right), \int_{t}^{t+d} \omega\right\rangle \\
= & \int_{t}^{t+d}\left\langle\partial\left(\gamma ; d_{1}, \ldots, d_{p}\right),(\omega)_{u}^{*}\right\rangle \mathbf{d} u \\
= & d\left\langle\partial\left(\gamma ; d_{1}, \ldots, d_{p}\right),(\omega)_{t}^{*}\right\rangle \\
= & d\left\langle\left(\gamma ; d_{1}, \ldots, d_{p}\right), \mathbf{d}(\omega)_{t}^{*}\right\rangle \\
= & d\left\langle\left(\gamma ; d_{1}, \ldots, d_{p}\right), \iota_{t}^{*} \mathbf{L}_{\frac{\partial}{\partial t}} \varphi^{*} \omega-(\mathbf{d} \omega)_{t}^{*}\right\rangle
\end{aligned}
$$

[by Proposition 3.12]

$$
\begin{aligned}
& =\left\langle\left(\gamma ; d_{1}, \ldots, d_{p}\right), d \iota_{t}^{*} \mathbf{L}_{\frac{\partial}{\partial t}} \varphi^{*} \omega\right\rangle-d\left\langle\left(\gamma ; d_{1}, \ldots, d_{p}\right),(\mathbf{d} \omega)_{t}^{*}\right\rangle \\
& =\left\langle\left(\gamma ; d_{1}, \ldots, d_{p}\right), \varphi_{t+d}^{*} \omega-\varphi_{t}^{*} \omega\right\rangle-\left\langle\left(\gamma ; d_{1}, \ldots, d_{p}\right), \int_{t}^{t+d} \mathbf{d} \omega\right\rangle \\
& =\left\langle\left(\gamma ; d_{1}, \ldots, d_{p}\right),-\int_{t}^{t+d} \mathbf{d} \omega-\varphi_{t}^{*} \omega+\varphi_{t+d}^{*} \omega\right\rangle .
\end{aligned}
$$


Corollary 3.14. Given $\omega \in \mathcal{A}^{p}(M)$ with $p$ being a positive integer, we have

$$
\mathbf{d} \int_{s}^{t} \omega=-\int_{s}^{t} \mathbf{d} \omega-\varphi_{s}^{*} \omega+\varphi_{t}^{*} \omega
$$

for any $s, t \in I$. In particular,

$$
\mathbf{d} \int \omega=-\int \mathbf{d} \omega-\varphi_{0}^{*} \omega+\varphi_{1}^{*} \omega .
$$

Proof. Let us define a function $F: I \rightarrow \mathbb{R}$ to be

$$
F(u)=\left\langle\left(\gamma ; d_{1}, \ldots, d_{p}\right), \mathbf{d} \int_{s}^{u} \omega+\int_{s}^{u} \mathbf{d} \omega+\varphi_{s}^{*} \omega-\varphi_{u}^{*} \omega\right\rangle
$$

for any $u \in I$. Then we have

$$
\begin{aligned}
& F(u+d)-F(u) \\
& =\left\langle\left(\gamma ; d_{1}, \ldots, d_{p}\right), \mathbf{d} \int_{u}^{u+d} \omega+\int_{u}^{u+d} \mathbf{d} \omega+\varphi_{u}^{*} \omega-\varphi_{u+d}^{*} \omega\right\rangle \\
& =0
\end{aligned}
$$

by dint of the above corollary, which implies that

$$
F^{\prime}(u)=0
$$

for any $u \in I$. Since

$$
F(s)=0
$$

holds trivially, we are done.

It is easy to see that

Proposition 3.15. Given $\omega \in \mathcal{A}^{p}(M)$ with $p$ being a positive integer and $s, s^{\prime}, t, t^{\prime} \in I$, we have

$$
\int_{s}^{t} \omega=\int_{s}^{s^{\prime}} \omega+\int_{s^{\prime}}^{t^{\prime}} \omega+\int_{t^{\prime}}^{t} \omega
$$

\section{ITERATED INTEGRALS}

Definition 4.1. Given $s_{1}, \ldots, s_{k}, t \in I$ and $\omega_{1} \in \mathcal{A}^{p_{1}}(M), \ldots, \omega_{k} \in \mathcal{A}^{p_{k}}(M)$ with $p_{1}, \ldots, p_{k}$ being positive integers, we define

$$
\int_{s_{1}, \ldots, s_{k}}^{t} \omega_{1} \ldots \omega_{k} \in \mathcal{A}^{p_{1}+\ldots+p_{k}-k}(\mathcal{P} M)
$$

by induction on $k$ to be

$$
\int_{s_{k}}^{t}\left(\left(\int_{s_{1}, \ldots, s_{k-1}}^{u} \omega_{1} \ldots \omega_{k-1}\right) \wedge\left(\omega_{k}\right)_{u}^{*}\right) \mathbf{d} u .
$$


By way of example, we have

$$
\int_{s_{1}, s_{2}}^{t} \omega_{1} \omega_{2}=\int_{s_{2}}^{t}\left(\left(\int_{s_{1}}^{u} \omega_{1}\right) \wedge\left(\omega_{2}\right)_{u}^{*}\right) \mathbf{d} u .
$$

Notation 4.2. Given $\omega_{1} \in \mathcal{A}^{p_{1}}(M), \ldots, \omega_{k} \in \mathcal{A}^{p_{k}}(M)$ with $p_{1}, \ldots, p_{k}$ being positive integers, we write

$$
\int \omega_{1} \ldots \omega_{k}
$$

for

$$
\int_{0, \ldots, 0}^{1} \omega_{1} \ldots \omega_{k} .
$$

Notation 4.3. Since the space $\mathcal{A}^{p}(\mathcal{P} M)$ with $p$ being a natural number is a Euclidean $\mathbb{R}$-module, any mapping $\widetilde{\omega}: I \rightarrow \mathcal{A}^{p}(\mathcal{P} M)$ and any $t \in I$ give rise to a unique $\mathbf{D}_{t} \widetilde{\omega} \in \mathcal{A}^{p}(\mathcal{P} M)$ such that

$$
\widetilde{\omega}(t+d)-\widetilde{\omega}(t)=d \mathbf{D}_{t} \widetilde{\omega}
$$

for any $d \in D$.

It is easy to see that

Proposition 4.4. Suppose that we are given $\omega \in \mathcal{A}^{p}(M)$ with $p$ being a positive integer and $t \in I$. Let $\widetilde{\omega}: I \rightarrow \mathcal{A}^{p}(\mathcal{P} M)$ be the mapping $s \in I \mapsto \varphi_{s}^{*} \omega$. Then we have

$$
\mathbf{D}_{t}(\widetilde{\omega})=\iota_{t}^{*} \mathbf{L}_{\frac{\partial}{\partial t}} \varphi^{*} \omega .
$$

The following two are no other than variants of the fundametal theorem in calculus.

Proposition 4.5. Given $s, t \in I$ and a mapping $\widetilde{\omega}: I \rightarrow \mathcal{A}^{p}(\mathcal{P} M)$ with $p$ being a natural number, we have

$$
\mathbf{D}_{t}\left(\int_{s}^{t} \widetilde{\omega}(u) \mathbf{d} u\right)=\widetilde{\omega}(t) .
$$

Proposition 4.6. Given $s, t \in I$ and a mapping $\widetilde{\omega}: I \rightarrow \mathcal{A}^{p}(\mathcal{P} M)$ with $p$ being a natural number, we have

$$
\int_{s}^{t} \mathbf{D}_{u} \widetilde{\omega}(u) \mathbf{d} u=\widetilde{\omega}(t)-\widetilde{\omega}(s) .
$$

It is easy to see that

Proposition 4.7. Given mappings $\widetilde{\omega}_{1}: I \rightarrow \mathcal{A}^{p_{1}}(\mathcal{P} M)$ and $\widetilde{\omega}_{2}: I \rightarrow \mathcal{A}^{p_{2}}(\mathcal{P} M)$ with $p_{1}$ and $p_{2}$ being natural numbers, we have

$$
\begin{aligned}
& \mathbf{D}_{t}\left(\widetilde{\omega}_{1}(t) \wedge \widetilde{\omega}_{2}(t)\right) \\
= & \mathbf{D}_{t} \widetilde{\omega}_{1}(t) \wedge \widetilde{\omega}_{2}(t)+\widetilde{\omega}_{1}(t) \wedge \mathbf{D}_{t} \widetilde{\omega}_{2}(t)
\end{aligned}
$$

for any $t \in I$. 
Lemma 4.8. Let $s_{1}, s_{2}, t \in I$. Given $\omega_{1} \in \mathcal{A}^{p_{1}}(M)$ and $\omega_{2} \in \mathcal{A}^{p_{2}}(M)$ with $p_{1}$ and $p_{2}$ being positive integers, we have

$$
\begin{aligned}
& \int_{s_{2}}^{t}\left(\omega_{1}\right)_{u}^{*} \wedge \varphi_{u}^{*} \omega_{2} \mathbf{d} u+\int_{s_{2}}^{t}\left(\int_{s_{1}}^{u} \omega_{1}\right) \wedge\left(\iota_{u}^{*} \mathbf{L}_{\frac{\partial}{\partial t}} \varphi^{*} \omega_{2}\right) \mathbf{d} u \\
= & \left(\int_{s_{1}}^{t} \omega_{1}\right) \wedge \varphi_{t}^{*} \omega_{2}-\left(\int_{s_{1}}^{s_{2}} \omega_{1}\right) \wedge \varphi_{s_{2}}^{*} \omega_{2} .
\end{aligned}
$$

Proof. We have

$$
\mathbf{D}_{u}\left(\left(\int_{s_{1}}^{u} \omega_{1}\right) \wedge \varphi_{u}^{*} \omega_{2}\right)=\left(\omega_{1}\right)_{u}^{*} \wedge \varphi_{u}^{*} \omega_{2}+\left(\int_{s_{1}}^{u} \omega_{1}\right) \wedge\left(\iota_{u}^{*} \mathbf{L}_{\frac{\partial}{\partial t}} \varphi^{*} \omega_{2}\right)
$$

so that the desired formula follows by dint of Proposition 4.6.

Theorem 4.9. Let $s_{1}, s_{2}, t \in I$. Given $\omega_{1} \in \mathcal{A}^{p_{1}}(M)$ and $\omega_{2} \in \mathcal{A}^{p_{2}}(M)$ with $p_{1}$ and $p_{2}$ being positive integers, we have

$$
\begin{aligned}
& \mathbf{d} \int_{s_{1}, s_{2}}^{t} \omega_{1} \omega_{2} \\
= & -\int_{s_{1}, s_{2}}^{t}\left(\mathbf{d} \omega_{1}\right) \omega_{2}+(-1)^{p_{1}} \int_{s_{1}, s_{2}}^{t} \omega_{1}\left(\mathbf{d} \omega_{2}\right)+(-1)^{p_{1}} \int_{s_{2}}^{t} \omega_{1} \wedge \omega_{2} \\
- & \varphi_{s_{1}}^{*} \omega_{1} \wedge \int_{s_{2}}^{t} \omega_{2}+(-1)^{p_{1}}\left(\int_{s_{1}}^{s_{2}} \omega_{1}\right) \wedge \varphi_{s_{2}}^{*} \omega_{2}-(-1)^{p_{1}}\left(\int_{s_{1}}^{t} \omega_{1}\right) \wedge \varphi_{t}^{*} \omega_{2} .
\end{aligned}
$$

In particular, we have

$$
\begin{aligned}
& \mathbf{d} \int \omega_{1} \omega_{2} \\
= & -\int\left(\mathbf{d} \omega_{1}\right) \omega_{2}+(-1)^{p_{1}} \int \omega_{1}\left(\mathbf{d} \omega_{2}\right)+(-1)^{p_{1}} \int \omega_{1} \wedge \omega_{2} \\
- & \varphi_{0}^{*} \omega_{1} \wedge \int \omega_{2}-(-1)^{p_{1}}\left(\int \omega_{1}\right) \wedge \varphi_{1}^{*} \omega_{2} .
\end{aligned}
$$

Proof. We have

$$
\begin{aligned}
& \mathbf{d} \int_{s_{1}, s_{2}}^{t} \omega_{1} \omega_{2} \\
= & \mathbf{d} \int_{s_{2}}^{t}\left(\int_{s_{1}}^{u} \omega_{1}\right) \wedge\left(\omega_{2}\right)_{u}^{*} \mathbf{d} u \\
= & \int_{s_{2}}^{t}\left\{\left(\mathbf{d} \int_{s_{1}}^{u} \omega_{1}\right) \wedge\left(\omega_{2}\right)_{u}^{*}+(-1)^{p_{1}-1}\left(\int_{s_{1}}^{u} \omega_{1}\right) \wedge \mathbf{d}\left(\omega_{2}\right)_{u}^{*}\right\} \mathbf{d} u
\end{aligned}
$$

[by Proposition 3.9]

$$
\begin{aligned}
& =\int_{s_{2}}^{t}\left(\mathbf{d} \int_{s_{1}}^{u} \omega_{1}\right) \wedge\left(\omega_{2}\right)_{u}^{*} \mathbf{d} u+(-1)^{p_{1}-1} \int_{s_{2}}^{t}\left(\int_{s_{1}}^{u} \omega_{1}\right) \wedge \mathbf{d}\left(\omega_{2}\right)_{u}^{*} \mathbf{d} u \\
& =\int_{s_{2}}^{t}\left(-\int_{s_{1}}^{u} \mathbf{d} \omega_{1}-\varphi_{s_{1}}^{*} \omega_{1}+\varphi_{u}^{*} \omega_{1}\right) \wedge\left(\omega_{2}\right)_{u}^{*} \mathbf{d} u
\end{aligned}
$$


$+(-1)^{p_{1}-1} \int_{s_{2}}^{t}\left(\int_{s_{1}}^{u} \omega_{1}\right) \wedge\left(\iota_{u}^{*} \mathbf{L}_{\frac{\partial}{\partial t}} \varphi^{*} \omega_{2}-\left(\mathbf{d} \omega_{2}\right)_{u}^{*}\right) \mathbf{d} u$

[by Proposition 3.12 and Corollary 3.14]

$$
\begin{aligned}
& =-\int_{s_{1}, s_{2}}^{t}\left(\mathbf{d} \omega_{1}\right) \omega_{2}+(-1)^{p_{1}} \int_{s_{1}, s_{2}}^{t} \omega_{1}\left(\mathbf{d} \omega_{2}\right)-\left(\varphi_{s_{1}}^{*} \omega_{1}\right) \wedge\left(\int_{s_{2}}^{t}\left(\omega_{2}\right)_{u}^{*} \mathbf{d} u\right) \\
& +\int_{s_{2}}^{t}\left(\varphi_{u}^{*} \omega_{1}\right) \wedge\left(\omega_{2}\right)_{u}^{*} \mathbf{d} u-(-1)^{p_{1}} \int_{s_{2}}^{t}\left(\int_{s_{1}}^{u} \omega_{1}\right) \wedge\left(\iota_{u}^{*} \mathbf{L}_{\frac{\partial}{\partial t}} \varphi^{*} \omega_{2}\right) \mathbf{d} u \\
& =-\int_{s_{1}, s_{2}}^{t}\left(\mathbf{d} \omega_{1}\right) \omega_{2}+(-1)^{p_{1}} \int_{s_{1}, s_{2}}^{t} \omega_{1}\left(\mathbf{d} \omega_{2}\right) \\
& -\left(\varphi_{s_{1}}^{*} \omega_{1}\right) \wedge\left(\int_{s_{2}}^{t} \omega_{2}\right)+\int_{s_{2}}^{t}\left(\varphi_{u}^{*} \omega_{1}\right) \wedge\left(\omega_{2}\right)_{u}^{*} \mathbf{d} u \\
& -(-1)^{p_{1}}\left\{\left(\int_{s_{1}}^{t} \omega_{1}\right) \wedge \varphi_{t}^{*} \omega_{2}-\left(\int_{s_{1}}^{s_{2}} \omega_{1}\right) \wedge \varphi_{s_{2}}^{*} \omega_{2}-\int_{s_{2}}^{t}\left(\omega_{1}\right)_{u}^{*} \wedge \varphi_{u}^{*} \omega_{2} \mathbf{d} u\right\}
\end{aligned}
$$

[by Lemma 4.8]

$$
\begin{aligned}
& =-\int_{s_{1}, s_{2}}^{t}\left(\mathbf{d} \omega_{1}\right) \omega_{2}+(-1)^{p_{1}} \int_{s_{1}, s_{2}}^{t} \omega_{1}\left(\mathbf{d} \omega_{2}\right) \\
& +\left\{\int_{s_{2}}^{t}\left(\varphi_{u}^{*} \omega_{1}\right) \wedge\left(\omega_{2}\right)_{u}^{*} \mathbf{d} u+(-1)^{p_{1}} \int_{s_{2}}^{t}\left(\omega_{1}\right)_{u}^{*} \wedge \varphi_{u}^{*} \omega_{2} \mathbf{d} u\right\} \\
& -\left(\varphi_{s_{1}}^{*} \omega_{1}\right) \wedge\left(\int_{s_{2}}^{t} \omega_{2}\right)+(-1)^{p_{1}}\left(\int_{s_{1}}^{s_{2}} \omega_{1}\right) \wedge \varphi_{s_{2}}^{*} \omega_{2}-(-1)^{p_{1}}\left(\int_{s_{1}}^{t} \omega_{1}\right) \wedge \varphi_{t}^{*} \omega_{2} \\
& =-\int_{s_{1}, s_{2}}^{t}\left(\mathbf{d} \omega_{1}\right) \omega_{2}+(-1)^{p_{1}} \int_{s_{1}, s_{2}}^{t} \omega_{1}\left(\mathbf{d} \omega_{2}\right)+(-1)^{p_{1}} \int_{s_{2}}^{t} \omega_{1} \wedge \omega_{2} \\
& -\left(\varphi_{s_{1}}^{*} \omega_{1}\right) \wedge\left(\int_{s_{2}}^{t} \omega_{2}\right)+(-1)^{p_{1}}\left(\int_{s_{1}}^{s_{2}} \omega_{1}\right) \wedge \varphi_{s_{2}}^{*} \omega_{2}-(-1)^{p_{1}}\left(\int_{s_{1}}^{t} \omega_{1}\right) \wedge \varphi_{t}^{*} \omega_{2} .
\end{aligned}
$$

Lemma 4.10. Let $s_{1}, s_{2}, s_{3}, t \in I$. Given $\omega_{1} \in \mathcal{A}^{p_{1}}(M), \omega_{2} \in \mathcal{A}^{p_{2}}(M)$ and $\omega_{3} \in \mathcal{A}^{p_{3}}(M)$ with $p_{1}, p_{2}$ and $p_{3}$ being positive integers, we have

$$
\begin{aligned}
& \int_{s_{3}}^{t}\left(\int_{s_{1}}^{u} \omega_{1}\right) \wedge\left(\omega_{2}\right)_{u}^{*} \wedge \varphi_{u}^{*} \omega_{3} \mathbf{d} u+\int_{s_{3}}^{t}\left(\int_{s_{1}, s_{2}}^{u} \omega_{1} \omega_{2}\right) \wedge\left(\iota_{u}^{*} \mathbf{L}_{\frac{\partial}{\partial t}} \varphi^{*} \omega_{3}\right) \mathbf{d} u \\
= & \left(\int_{s_{1}, s_{2}}^{t} \omega_{1} \omega_{2}\right) \wedge \varphi_{t}^{*} \omega_{3}-\left(\int_{s_{1}, s_{2}}^{s_{3}} \omega_{1} \omega_{2}\right) \wedge \varphi_{s_{3}}^{*} \omega_{3} .
\end{aligned}
$$

Proof. We have

$$
\mathbf{D}_{u}\left(\left(\int_{s_{1}, s_{2}}^{u} \omega_{1} \omega_{2}\right) \wedge \varphi_{u}^{*} \omega_{3}\right)
$$




$$
=\left(\int_{s_{1}}^{u} \omega_{1}\right) \wedge\left(\omega_{2}\right)_{u}^{*} \wedge \varphi_{u}^{*} \omega_{3}+\left(\int_{s_{1}, s_{2}}^{u} \omega_{1} \omega_{2}\right) \wedge\left(\iota_{u}^{*} \mathbf{L}_{\frac{\partial}{\partial t}} \varphi^{*} \omega_{3}\right)
$$

Theorem 4.11. Let $s_{1}, s_{2}, s_{3}, t \in I$. Given $\omega_{1} \in \mathcal{A}^{p_{1}}(M), \omega_{2} \in \mathcal{A}^{p_{2}}(M)$ and $\omega_{3} \in \mathcal{A}^{p_{3}}(M)$ with $p_{1}, p_{2}$ and $p_{3}$ being positive integers, we have

$$
\begin{aligned}
& \mathbf{d} \int_{s_{1}, s_{2}, s_{3}}^{t} \omega_{1} \omega_{2} \omega_{3} \\
= & -\int_{s_{1}, s_{2}, s_{3}}^{t}\left(\mathbf{d} \omega_{1}\right) \omega_{2} \omega_{3}+(-1)^{p_{1}} \int_{s_{1}, s_{2}, s_{3}}^{t} \omega_{1}\left(\mathbf{d} \omega_{2}\right) \omega_{3} \\
- & (-1)^{p_{1}+p_{2}} \int_{s_{1}, s_{2}, s_{3}}^{t} \omega_{1} \omega_{2}\left(\mathbf{d} \omega_{3}\right) \\
+ & (-1)^{p_{1}} \int_{s_{2}, s_{3}}^{t}\left(\omega_{1} \wedge \omega_{2}\right) \omega_{3}-(-1)^{p_{1}+p_{2}} \int_{s_{1}, s_{3}}^{t} \omega_{1}\left(\omega_{2} \wedge \omega_{3}\right)-\varphi_{s_{1}}^{*} \omega_{1} \wedge \int_{s_{2}, s_{3}}^{t} \omega_{2} \omega_{3} \\
+ & (-1)^{p_{1}}\left(\int_{s_{1}}^{s_{2}} \omega_{1}\right) \wedge\left(\varphi_{s_{2}}^{*} \omega_{2}\right) \wedge\left(\int_{s_{3}}^{t} \omega_{3}\right)-(-1)^{p_{1}+p_{2}}\left(\int_{s_{1}, s_{2}}^{s_{3}} \omega_{1} \omega_{2}\right) \wedge \varphi_{s_{3}}^{*} \omega_{3} \\
+ & (-1)^{p_{1}+p_{2}}\left(\int_{s_{1}, s_{2}}^{t} \omega_{1} \omega_{2}\right) \wedge \varphi_{t}^{*} \omega_{3} .
\end{aligned}
$$

In particular, we have

$$
\begin{aligned}
& \mathbf{d} \int \omega_{1} \omega_{2} \omega_{3} \\
= & -\int\left(\mathbf{d} \omega_{1}\right) \omega_{2} \omega_{3}+(-1)^{p_{1}} \int \omega_{1}\left(\mathbf{d} \omega_{2}\right) \omega_{3}-(-1)^{p_{1}+p_{2}} \int \omega_{1} \omega_{2}\left(\mathbf{d} \omega_{3}\right) \\
+ & (-1)^{p_{1}} \int\left(\omega_{1} \wedge \omega_{2}\right) \omega_{3}-(-1)^{p_{1}+p_{2}} \int \omega_{1}\left(\omega_{2} \wedge \omega_{3}\right) \\
- & \varphi_{0}^{*} \omega_{1} \wedge \int \omega_{2} \omega_{3}+(-1)^{p_{1}+p_{2}}\left(\int \omega_{1} \omega_{2}\right) \wedge \varphi_{1}^{*} \omega_{3} .
\end{aligned}
$$

Proof. We have

$$
\begin{aligned}
& \mathbf{d} \int_{s_{1}, s_{2}, s_{3}}^{t} \omega_{1} \omega_{2} \omega_{3} \\
= & \mathbf{d} \int_{s_{3}}^{t}\left(\int_{s_{1}, s_{2}}^{u} \omega_{1} \omega_{2}\right) \wedge\left(\omega_{3}\right)_{u}^{*} \mathbf{d} u \\
= & \int_{s_{3}}^{t}\left\{\left(\mathbf{d} \int_{s_{1}, s_{2}}^{u} \omega_{1} \omega_{2}\right) \wedge\left(\omega_{3}\right)_{u}^{*}+(-1)^{p_{1}+p_{2}}\left(\int_{s_{1}, s_{2}}^{u} \omega_{1} \omega_{2}\right) \wedge \mathbf{d}\left(\omega_{3}\right)_{u}^{*}\right\} \mathbf{d} u
\end{aligned}
$$

[by Proposition 3.9]

$$
=\int_{s_{3}}^{t}\left(\mathbf{d} \int_{s_{1}, s_{2}}^{u} \omega_{1} \omega_{2}\right) \wedge\left(\omega_{3}\right)_{u}^{*} \mathbf{d} u+(-1)^{p_{1}+p_{2}} \int_{s_{3}}^{t}\left(\int_{s_{1}, s_{2}}^{u} \omega_{1} \omega_{2}\right) \wedge \mathbf{d}\left(\omega_{3}\right)_{u}^{*} \mathbf{d} u
$$




$$
\begin{gathered}
\int_{s_{3}}^{t}\left(\begin{array}{c}
-\int_{s_{1}, s_{2}}^{u}\left(\mathbf{d} \omega_{1}\right) \omega_{2}+(-1)^{p_{1}} \int_{s_{1}, s_{2}}^{u} \omega_{1}\left(\mathbf{d} \omega_{2}\right)+(-1)^{p_{1}} \int_{s_{2}}^{u} \omega_{1} \wedge \omega_{2} \\
-\varphi_{s_{1}}^{*} \omega_{1} \wedge \int_{s_{2}}^{u} \omega_{2}+(-1)^{p_{1}}\left(\int_{s_{1}}^{s_{2}} \omega_{1}\right) \wedge \varphi_{s_{2}}^{*} \omega_{2} \\
-(-1)^{p_{1}}\left(\int_{s_{1}}^{u} \omega_{1}\right) \wedge \varphi_{u}^{*} \omega_{2}
\end{array}\right) \\
\wedge\left(\omega_{3}\right)_{u}^{*} \mathbf{d} u+(-1)^{p_{1}+p_{2}} \int_{s_{3}}^{t}\left(\int_{s_{1}, s_{2}}^{u} \omega_{1} \omega_{2}\right) \wedge\left(\iota_{u}^{*} \mathbf{L}_{\frac{\partial}{\partial t}} \varphi^{*} \omega_{3}-\left(\mathbf{d} \omega_{3}\right)_{u}^{*}\right) \mathbf{d} u
\end{gathered}
$$

[by Theorem 4.9 and Proposition 3.12]

$$
\begin{aligned}
& =-\int_{s_{1}, s_{2}, s_{3}}^{t}\left(\mathbf{d} \omega_{1}\right) \omega_{2} \omega_{3}+(-1)^{p_{1}} \int_{s_{1}, s_{2}, s_{3}}^{t} \omega_{1}\left(\mathbf{d} \omega_{2}\right) \omega_{3} \\
& -(-1)^{p_{1}+p_{2}} \int_{s_{1}, s_{2}, s_{3}}^{t} \omega_{1} \omega_{2}\left(\mathbf{d} \omega_{3}\right)+(-1)^{p_{1}} \int_{s_{2}, s_{3}}^{t}\left(\omega_{1} \wedge \omega_{2}\right) \omega_{3}-\varphi_{s_{1}}^{*} \omega_{1} \wedge \int_{s_{2}, s_{3}}^{t} \omega_{2} \omega_{3} \\
& +(-1)^{p_{1}}\left(\int_{s_{1}}^{s_{2}} \omega_{1}\right) \wedge \varphi_{s_{2}}^{*} \omega_{2} \wedge \int_{s_{3}}^{t} \omega_{3}-(-1)^{p_{1}} \int_{s_{3}}^{t}\left(\int_{s_{1}}^{u} \omega_{1}\right) \wedge \varphi_{u}^{*} \omega_{2} \wedge\left(\omega_{3}\right)_{u}^{*} \mathbf{d} u \\
& +(-1)^{p_{1}+p_{2}} \int_{s_{3}}^{t}\left(\int_{s_{1}, s_{2}}^{u} \omega_{1} \omega_{2}\right) \wedge\left(\iota_{u}^{*} \mathbf{L}_{\frac{\partial}{\partial t}} \varphi^{*} \omega_{3}\right) \mathbf{d} u \\
& =-\int_{s_{1}, s_{2}, s_{3}}^{t}\left(\mathbf{d} \omega_{1}\right) \omega_{2} \omega_{3}+(-1)^{p_{1}} \int_{s_{1}, s_{2}, s_{3}}^{t} \omega_{1}\left(\mathbf{d} \omega_{2}\right) \omega_{3} \\
& -(-1)^{p_{1}+p_{2}} \int_{s_{1}, s_{2}, s_{3}}^{t} \omega_{1} \omega_{2}\left(\mathbf{d} \omega_{3}\right)+(-1)^{p_{1}} \int_{s_{2}, s_{3}}^{t}\left(\omega_{1} \wedge \omega_{2}\right) \omega_{3}-\varphi_{s_{1}}^{*} \omega_{1} \wedge \int_{s_{2}, s_{3}}^{t} \omega_{2} \omega_{3} \\
& +(-1)^{p_{1}}\left(\int_{s_{1}}^{s_{2}} \omega_{1}\right) \wedge \varphi_{s_{2}}^{*} \omega_{2} \wedge \int_{s_{3}}^{t} \omega_{3}-(-1)^{p_{1}} \int_{s_{3}}^{t}\left(\int_{s_{1}}^{u} \omega_{1}\right) \wedge \varphi_{u}^{*} \omega_{2} \wedge\left(\omega_{3}\right)_{u}^{*} \mathbf{d} u \\
& +(-1)^{p_{1}+p_{2}}\left\{\begin{array}{c}
-\int_{s_{3}}^{t}\left(\int_{s_{1}}^{u} \omega_{1}\right) \wedge\left(\omega_{2}\right)_{u}^{*} \wedge \varphi_{u}^{*} \omega_{3} \mathbf{d} u \\
+\left(\int_{s_{1}, s_{2}}^{t} \omega_{1} \omega_{2}\right) \wedge \varphi_{t}^{*} \omega_{3}-\left(\int_{s_{1}, s_{2}}^{s_{3}} \omega_{1} \omega_{2}\right) \wedge \varphi_{s_{3}}^{*} \omega_{3}
\end{array}\right\}
\end{aligned}
$$

[by Lemma 4.10]

$$
\begin{aligned}
& =-\int_{s_{1}, s_{2}, s_{3}}^{t}\left(\mathbf{d} \omega_{1}\right) \omega_{2} \omega_{3}+(-1)^{p_{1}} \int_{s_{1}, s_{2}, s_{3}}^{t} \omega_{1}\left(\mathbf{d} \omega_{2}\right) \omega_{3} \\
& -(-1)^{p_{1}+p_{2}} \int_{s_{1}, s_{2}, s_{3}}^{t} \omega_{1} \omega_{2}\left(\mathbf{d} \omega_{3}\right)+(-1)^{p_{1}} \int_{s_{2}, s_{3}}^{t}\left(\omega_{1} \wedge \omega_{2}\right) \omega_{3} \\
& -(-1)^{p_{1}+p_{2}}\left(\begin{array}{c}
\int_{s_{3}}^{t}\left(\int_{s_{1}}^{u} \omega_{1}\right) \wedge\left(\omega_{2}\right)_{u}^{*} \wedge \varphi_{u}^{*} \omega_{3} \mathbf{d} u \\
+(-1)^{p_{2}} \int_{s_{3}}^{t}\left(\int_{s_{1}}^{u} \omega_{1}\right) \wedge \varphi_{u}^{*} \omega_{2} \wedge\left(\omega_{3}\right)_{u}^{*} \mathbf{d} u
\end{array}\right) \\
& -\varphi_{s_{1}}^{*} \omega_{1} \wedge \int_{s_{2}, s_{3}}^{t} \omega_{2} \omega_{3}+(-1)^{p_{1}}\left(\int_{s_{1}}^{s_{2}} \omega_{1}\right) \wedge\left(\varphi_{s_{2}}^{*} \omega_{2}\right) \wedge\left(\int_{s_{3}}^{t} \omega_{3}\right) \\
& +(-1)^{p_{1}+p_{2}}\left(\int_{s_{1}, s_{2}}^{t} \omega_{1} \omega_{2}\right) \wedge \varphi_{t}^{*} \omega_{3}-(-1)^{p_{1}+p_{2}}\left(\int_{s_{1}, s_{2}}^{s_{3}} \omega_{1} \omega_{2}\right) \wedge \varphi_{s_{3}}^{*} \omega_{3}
\end{aligned}
$$




$$
\begin{aligned}
& -\int_{s_{1}, s_{2}, s_{3}}^{t}\left(\mathbf{d} \omega_{1}\right) \omega_{2} \omega_{3}+(-1)^{p_{1}} \int_{s_{1}, s_{2}, s_{3}}^{t} \omega_{1}\left(\mathbf{d} \omega_{2}\right) \omega_{3} \\
& -(-1)^{p_{1}+p_{2}} \int_{s_{1}, s_{2}, s_{3}}^{t} \omega_{1} \omega_{2}\left(\mathbf{d} \omega_{3}\right)+(-1)^{p_{1}} \int_{s_{2}, s_{3}}^{t}\left(\omega_{1} \wedge \omega_{2}\right) \omega_{3} \\
& -(-1)^{p_{1}+p_{2}} \int_{s_{1}, s_{3}}^{t} \omega_{1}\left(\omega_{2} \wedge \omega_{3}\right)-\varphi_{s_{1}}^{*} \omega_{1} \wedge \int_{s_{2}, s_{3}}^{t} \omega_{2} \omega_{3} \\
& +(-1)^{p_{1}}\left(\int_{s_{1}}^{s_{2}} \omega_{1}\right) \wedge\left(\varphi_{s_{2}}^{*} \omega_{2}\right) \wedge\left(\int_{s_{3}}^{t} \omega_{3}\right)+(-1)^{p_{1}+p_{2}}\left(\int_{s_{1}, s_{2}}^{t} \omega_{1} \omega_{2}\right) \wedge \varphi_{t}^{*} \omega_{3} \\
& -(-1)^{p_{1}+p_{2}}\left(\int_{s_{1}, s_{2}}^{s_{3}} \omega_{1} \omega_{2}\right) \wedge \varphi_{s_{3}}^{*} \omega_{3} .
\end{aligned}
$$

In general, we have

Theorem 4.12. Let $s_{1}, \ldots, s_{k}, t \in I$. Given $\omega_{i} \in \mathcal{A}^{p_{i}}(M)$ with $p_{i}$ being a positive integer $(1 \leq i \leq k)$, we have

$$
\begin{aligned}
& \mathbf{d} \int_{s_{1}, \ldots, s_{3}}^{t} \omega_{1} \ldots \omega_{k} \\
= & \sum_{i=1}^{k}(-1)^{i+p_{1}+\ldots+p_{i-1}} \int_{s_{1}, \ldots, s_{k}}^{t} \omega_{1} \ldots \omega_{i-1}\left(\mathbf{d} \omega_{i}\right) \omega_{i+1} \ldots \omega_{k} \\
+ & \sum_{i=1}^{k-1}(-1)^{i+p_{1}+\ldots+p_{i}+1} \int_{s_{1}, \ldots, s_{k}}^{t} \omega_{1} \ldots \omega_{i-1}\left(\omega_{i} \wedge \omega_{i+1}\right) \omega_{i+2} \ldots \omega_{k} \\
+ & \sum_{i=1}^{k}(-1)^{i+p_{1}+\ldots+p_{i-1}}\left(\int_{s_{1}, \ldots, s_{i-1}}^{s_{i}} \omega_{1} \ldots \omega_{i-1}\right) \wedge\left(\varphi_{s_{i}}^{*} \omega_{i}\right) \wedge\left(\int_{s_{i+1}, \ldots, s_{k}}^{t} \omega_{i+1} \ldots \omega_{k}\right) \\
+ & (-1)^{p_{1}+\ldots+p_{k-1}+k+1}\left(\int_{s_{1}, \ldots, s_{k-1}}^{t} \omega_{1} \ldots \omega_{k-1}\right) \wedge\left(\varphi_{t}^{*} \omega_{k}\right) .
\end{aligned}
$$

In particular, we have

$$
\begin{aligned}
& \mathbf{d} \int \omega_{1} \ldots \omega_{k} \\
- & \sum_{i=1}^{k}(-1)^{i+p_{1}+\ldots+p_{i-1}} \int \omega_{1} \ldots \omega_{i-1}\left(\mathbf{d} \omega_{i}\right) \omega_{i+1} \ldots \omega_{k} \\
+ & \sum_{i=1}^{k-1}(-1)^{i+p_{1}+\ldots+p_{i}+1} \int \omega_{1} \ldots \omega_{i-1}\left(\omega_{i} \wedge \omega_{i+1}\right) \omega_{i+2} \ldots \omega_{k} \\
- & \left(\varphi_{0}^{*} \omega_{1}\right) \wedge\left(\int \omega_{2} \ldots \omega_{k}\right) \\
+ & (-1)^{p_{1}+\ldots+p_{k-1}+k+1}\left(\int \omega_{1} \ldots \omega_{k-1}\right) \wedge\left(\varphi_{1}^{*} \omega_{k}\right) .
\end{aligned}
$$


Corollary 4.13. Given $\omega_{i} \in \mathcal{A}^{p_{i}}(M)(0 \leq i \leq k+1)$ with $p_{i}$ being a positive integer $(1 \leq i \leq k)$ and $p_{0}$ and $p_{k+1}$ being non-negative integers, we have

$$
\begin{aligned}
& \mathbf{d}\left(\left(\varphi_{0}^{*} \omega_{0}\right) \wedge\left(\int \omega_{1} \ldots \omega_{k}\right) \wedge\left(\varphi_{1}^{*} \omega_{k+1}\right)\right) \\
= & \left(\varphi_{0}^{*}\left(\mathbf{d} \omega_{0}\right)\right) \wedge\left(\int \omega_{1} \ldots \omega_{k}\right) \wedge\left(\varphi_{1}^{*} \omega_{k+1}\right) \\
+ & \sum_{i=1}^{k}(-1)^{i+p_{0}+p_{1}+\ldots+p_{i-1}}\left(\varphi_{0}^{*} \omega_{0}\right) \wedge\left(\int \omega_{1} \ldots \omega_{i-1}\left(\mathbf{d} \omega_{i}\right) \omega_{i+1} \ldots \omega_{k}\right) \wedge\left(\varphi_{1}^{*} \omega_{k+1}\right) \\
+ & \sum_{i=1}^{k-1}(-1)^{i+p_{0}+p_{1}+\ldots+p_{i}+1}\left(\varphi_{0}^{*} \omega_{0}\right) \\
& \wedge\left(\int \omega_{1} \ldots \omega_{i-1}\left(\omega_{i} \wedge \omega_{i+1}\right) \omega_{i+2} \ldots \omega_{k}\right) \wedge\left(\varphi_{1}^{*} \omega_{k+1}\right) \\
- & (-1)^{p_{0}}\left(\varphi_{0}^{*}\left(\omega_{0} \wedge \omega_{1}\right)\right) \wedge\left(\int \omega_{2} \ldots \omega_{k}\right) \wedge\left(\varphi_{1}^{*} \omega_{k+1}\right) \\
+ & (-1)^{p_{0}+p_{1}+\ldots+p_{k-1}+k+1}\left(\int \omega_{1} \ldots \omega_{k-1}\right) \wedge\left(\varphi_{1}^{*}\left(\omega_{k} \wedge \omega_{k+1}\right)\right) \\
+ & (-1)^{p_{0}+p_{1}+\ldots+p_{k}+k}\left(\varphi_{0}^{*} \omega_{0}\right) \wedge\left(\int \omega_{1} \ldots \omega_{k}\right) \wedge\left(\varphi_{1}^{*} \mathbf{d} \omega_{k+1}\right)
\end{aligned}
$$

so that the graded submodule of the de Rham module on $\mathcal{P} M$ linearly generated by differential forms of the form $\left(\varphi_{0}^{*} \omega_{0}\right) \wedge\left(\int \omega_{1} \ldots \omega_{k}\right) \wedge\left(\varphi_{1}^{*} \omega_{k+1}\right)$ is closed under exterior differentiation, constituting a subcomplex of the de Rham complex $\mathcal{A}(\mathcal{P} M)$.

Definition 4.14. The subcomplex in the above corollary is called the Hochschild complex associated with the de Rham complex $\mathcal{A}(M)$.

We conclude this section with the following simple proposition.

Proposition 4.15. Let $s_{1}, s_{2}, s_{1}^{\prime}, s_{2}^{\prime}, t, t^{\prime} \in I$. Let $\omega_{1} \in \mathcal{A}^{p_{1}}(M)$ and $\omega_{2} \in$ $\mathcal{A}^{p_{2}}(M)$. We have

$$
\begin{aligned}
& \int_{s_{1}, s_{2}}^{t} \omega_{1} \omega_{2}=\int_{s_{1}^{\prime}, s_{2}}^{t} \omega_{1} \omega_{2}+\left(\int_{s_{1}}^{s_{1}^{\prime}} \omega_{1}\right) \wedge\left(\int_{s_{2}}^{t} \omega_{2}\right), \\
& \int_{s_{1}, s_{2}}^{t} \omega_{1} \omega_{2}=\int_{s_{1}, s_{2}}^{s_{2}^{\prime}} \omega_{1} \omega_{2}+\int_{s_{1}, s_{2}^{\prime}}^{t} \omega_{1} \omega_{2}, \\
& \int_{s_{1}, s_{2}}^{t} \omega_{1} \omega_{2}=\int_{s_{1}, s_{2}}^{t^{\prime}} \omega_{1} \omega_{2}+\int_{s_{1}, t^{\prime}}^{t} \omega_{1} \omega_{2} .
\end{aligned}
$$

Proof. Here we deal only with the first formula. We have

$$
\begin{aligned}
& \int_{s_{1}, s_{2}}^{t} \omega_{1} \omega_{2} \\
= & \int_{s_{2}}^{t}\left(\int_{s_{1}}^{u} \omega_{1}\right) \wedge\left(\omega_{2}\right)_{u}^{*} \mathbf{d} u
\end{aligned}
$$


138

H. NISHIMURA

$$
\begin{aligned}
= & \int_{s_{2}}^{t}\left(\int_{s_{1}^{\prime}}^{u} \omega_{1}+\int_{s_{1}}^{s_{1}^{\prime}} \omega_{1}\right) \wedge\left(\omega_{2}\right)_{u}^{*} \mathbf{d} u \\
= & \int_{s_{2}}^{t}\left(\int_{s_{1}^{\prime}}^{u} \omega_{1}\right) \wedge\left(\omega_{2}\right)_{u}^{*} \mathbf{d} u+\int_{s_{2}}^{t}\left(\int_{s_{1}}^{s_{1}^{\prime}} \omega_{1}\right) \wedge\left(\omega_{2}\right)_{u}^{*} \mathbf{d} u \\
= & \int_{s_{1}, s_{2}^{\prime}}^{t} \omega_{1} \omega_{2}+\left(\int_{s_{1}}^{s_{1}^{\prime}} \omega_{1}\right) \wedge\left(\int_{s_{2}}^{t} \omega_{2}\right) \\
& \int_{s_{1}, s_{2}}^{t} \omega_{1} \omega_{2} \\
= & \int_{s_{2}}^{t}\left(\int_{s_{1}}^{u} \omega_{1}\right) \wedge\left(\omega_{2}\right)_{u}^{*} \mathbf{d} u \\
= & \int_{s_{2}}^{s_{2}^{\prime}}\left(\int_{s_{1}}^{u} \omega_{1}\right) \wedge\left(\omega_{2}\right)_{u}^{*} \mathbf{d} u+\int_{s_{2}^{\prime}}^{t}\left(\int_{s_{1}}^{u} \omega_{1}\right) \wedge\left(\omega_{2}\right)_{u}^{*} \mathbf{d} u \\
= & \int_{s_{1}, s_{2}}^{s_{2}^{\prime}} \omega_{1} \omega_{2}+\int_{s_{1}, s_{2}^{\prime}}^{t} \omega_{1} \omega_{2} .
\end{aligned}
$$

Corollary 4.16.

$$
\begin{aligned}
& \int_{s_{1}, s_{2}}^{t} \omega_{1} \omega_{2} \\
= & \int_{s_{1}^{\prime}, s_{2}}^{s_{2}^{\prime}} \omega_{1} \omega_{2}+\int_{s_{1}^{\prime}, s_{2}^{\prime}}^{t^{\prime}} \omega_{1} \omega_{2}+\int_{s_{1}^{\prime}, t^{\prime}}^{t} \omega_{1} \omega_{2} \\
+ & \left(\int_{s_{1}}^{s_{1}^{\prime}} \omega_{1}\right) \wedge\left(\int_{s_{2}}^{s_{2}^{\prime}} \omega_{2}\right)+\left(\int_{s_{1}}^{s_{1}^{\prime}} \omega_{1}\right) \wedge\left(\int_{s_{2}^{\prime}}^{t^{\prime}} \omega_{2}\right)+\left(\int_{s_{1}}^{s_{1}^{\prime}} \omega_{1}\right) \wedge\left(\int_{t^{\prime}}^{t} \omega_{2}\right) .
\end{aligned}
$$

Proof. We have

$$
\begin{aligned}
& \int_{s_{1}, s_{2}}^{t} \omega_{1} \omega_{2} \\
= & \int_{s_{1}^{\prime}, s_{2}}^{t} \omega_{1} \omega_{2}+\left(\int_{s_{1}}^{s_{1}^{\prime}} \omega_{1}\right) \wedge\left(\int_{s_{2}}^{t} \omega_{2}\right) \\
= & \int_{s_{1}^{\prime}, s_{2}}^{s_{2}^{\prime}} \omega_{1} \omega_{2}+\int_{s_{1}^{\prime}, s_{2}^{\prime}}^{t} \omega_{1} \omega_{2} \\
+ & \left(\int_{s_{1}}^{s_{1}^{\prime}} \omega_{1}\right) \wedge\left(\int_{s_{2}}^{s_{2}^{\prime}} \omega_{2}\right)+\left(\int_{s_{1}}^{s_{1}^{\prime}} \omega_{1}\right) \wedge\left(\int_{s_{2}^{\prime}}^{t^{\prime}} \omega_{2}\right)+\left(\int_{s_{1}}^{s_{1}^{\prime}} \omega_{1}\right) \wedge\left(\int_{t^{\prime}}^{t} \omega_{2}\right) \\
= & \int_{s_{1}^{\prime}, s_{2}}^{s_{2}^{\prime}} \omega_{1} \omega_{2}+\int_{s_{1}^{\prime}, s_{2}^{\prime}}^{t^{\prime}} \omega_{1} \omega_{2}+\int_{s_{1}^{\prime}, t^{\prime}}^{t} \omega_{1} \omega_{2}
\end{aligned}
$$




$$
+\left(\int_{s_{1}}^{s_{1}^{\prime}} \omega_{1}\right) \wedge\left(\int_{s_{2}}^{s_{2}^{\prime}} \omega_{2}\right)+\left(\int_{s_{1}}^{s_{1}^{\prime}} \omega_{1}\right) \wedge\left(\int_{s_{2}^{\prime}}^{t^{\prime}} \omega_{2}\right)+\left(\int_{s_{1}}^{s_{1}^{\prime}} \omega_{1}\right) \wedge\left(\int_{t^{\prime}}^{t} \omega_{2}\right) .
$$

\section{Based Path Spaces}

Let us begin by fixing our notation.

Notation 5.1. Let $x_{1}, x_{2} \in M$. We denote by $\mathcal{P}_{x_{1}} M, \mathcal{P}^{x_{2}} M$ and $\mathcal{P}_{x_{1}}^{x_{2}} M$ the spaces

$$
\begin{aligned}
& \mathcal{P}_{x_{1}} M=\left\{\theta \in \mathcal{P} M \mid \theta(0)=x_{1}\right\}, \\
& \mathcal{P}^{x_{2}} M=\left\{\theta \in \mathcal{P} M \mid \theta(1)=x_{2}\right\}, \\
& \mathcal{P}_{x_{1}}^{x_{2}} M=\left\{\theta \in \mathcal{P} M \mid \theta(0)=x_{1} \text { and } \theta(1)=x_{2}\right\} .
\end{aligned}
$$

Notation 5.2. For simplicity, we use the same notation $\varphi: I \times \mathcal{P}_{x_{1}} M \rightarrow M$, $\varphi: I \times \mathcal{P}^{x_{2}} M \rightarrow M$ and $\varphi: I \times \mathcal{P}_{x_{1}}^{x_{2}} M \rightarrow M$ for the restrictions of $\varphi: I \times \mathcal{P} M \rightarrow M$.

We have the following three theorems as corollaries of Theorem 4.12.

Theorem 5.3. Let $x_{1} \in M$. Given $\omega_{i} \in \mathcal{A}^{p_{i}}(M)(1 \leq i \leq k+1)$ with $p_{i}$ being a positive integer $(1 \leq i \leq k)$ and $p_{k+1}$ being a non-negative integer, we have

$$
\begin{aligned}
& \mathbf{d}\left(\left(\int \omega_{1} \ldots \omega_{k}\right) \wedge\left(\varphi_{1}^{*} \omega_{k+1}\right)\right) \\
= & \left(\int \omega_{1} \ldots \omega_{k}\right) \wedge\left(\varphi_{1}^{*} \omega_{k+1}\right) \\
+ & \sum_{i=1}^{k}(-1)^{i+p_{1}+\ldots+p_{i-1}}\left(\int \omega_{1} \ldots \omega_{i-1}\left(\mathbf{d} \omega_{i}\right) \omega_{i+1} \ldots \omega_{k}\right) \wedge\left(\varphi_{1}^{*} \omega_{k+1}\right) \\
+ & \sum_{i=1}^{k-1}(-1)^{i+p_{1}+\ldots+p_{i}+1}\left(\int \omega_{1} \ldots \omega_{i-1}\left(\omega_{i} \wedge \omega_{i+1}\right) \omega_{i+2} \ldots \omega_{k}\right) \wedge\left(\varphi_{1}^{*} \omega_{k+1}\right) \\
+ & (-1)^{p_{1}+\ldots+p_{k-1}+k+1}\left(\int \omega_{1} \ldots \omega_{k-1}\right) \wedge\left(\varphi_{1}^{*}\left(\omega_{k} \wedge \omega_{k+1}\right)\right) \\
+ & (-1)^{p_{1}+\ldots+p_{k}+k}\left(\int \omega_{1} \ldots \omega_{k}\right) \wedge\left(\varphi_{1}^{*} \mathbf{d} \omega_{k+1}\right)
\end{aligned}
$$

so that the graded submodule of the de Rham module on $\mathcal{P}_{x_{1}} M$ linearly generated by differential forms of the form $\left(\int \omega_{1} \ldots \omega_{k}\right) \wedge\left(\varphi_{1}^{*} \omega_{k+1}\right)$ is closed under exterior differentiation, constituting a subcomplex of the de Rham complex $\mathcal{A}\left(\mathcal{P}_{x_{1}} M\right)$.

Theorem 5.4. Let $x_{2} \in M$. Given $\omega_{i} \in \mathcal{A}^{p_{i}}(M)(0 \leq i \leq k)$ with $p_{i}$ being a positive integer $(1 \leq i \leq k)$ and $p_{0}$ being a non-negative integer, we have

$$
\begin{aligned}
& \mathbf{d}\left(\left(\varphi_{0}^{*} \omega_{0}\right) \wedge\left(\int \omega_{1} \ldots \omega_{k}\right)\right) \\
= & \left(\varphi_{0}^{*}\left(\mathbf{d} \omega_{0}\right)\right) \wedge\left(\int \omega_{1} \ldots \omega_{k}\right)
\end{aligned}
$$




$$
\begin{aligned}
& +\sum_{i=1}^{k}(-1)^{i+p_{0}+p_{1}+\ldots+p_{i-1}}\left(\varphi_{0}^{*} \omega_{0}\right) \wedge\left(\int \omega_{1} \ldots \omega_{i-1}\left(\mathbf{d} \omega_{i}\right) \omega_{i+1} \ldots \omega_{k}\right) \\
& +\sum_{i=1}^{k-1}(-1)^{i+p_{0}+p_{1}+\ldots+p_{i}+1}\left(\varphi_{0}^{*} \omega_{0}\right) \wedge\left(\int \omega_{1} \ldots \omega_{i-1}\left(\omega_{i} \wedge \omega_{i+1}\right) \omega_{i+2} \ldots \omega_{k}\right) \\
& -(-1)^{p_{0}}\left(\varphi_{0}^{*}\left(\omega_{0} \wedge \omega_{1}\right)\right) \wedge\left(\int \omega_{2} \ldots \omega_{k}\right)
\end{aligned}
$$

so that the graded submodule of the de Rham module on $\mathcal{P}^{x_{2}} M$ linearly generated by differential forms of the form $\left(\varphi_{0}^{*} \omega_{0}\right) \wedge\left(\int \omega_{1} \ldots \omega_{k}\right)$ is closed under exterior differentiation, constituting a subcomplex of the de Rham complex $\mathcal{A}\left(\mathcal{P}^{x_{2}} M\right)$.

Theorem 5.5. Let $x_{1}, x_{2} \in M$. Given $\omega_{i} \in \mathcal{A}^{p_{i}}(M)(1 \leq i \leq k)$ with $p_{i}$ being a positive integer $(1 \leq i \leq k)$, we have

$$
\begin{aligned}
& \mathbf{d} \int \omega_{1} \ldots \omega_{k} \\
= & \sum_{i=1}^{k}(-1)^{p_{1}+\ldots+p_{i-1}+i} \int \omega_{1} \ldots \omega_{i-1}\left(\mathbf{d} \omega_{i}\right) \omega_{i+1} \ldots \omega_{k} \\
+ & \sum_{i=1}^{k-1}(-1)^{p_{1}+\ldots+p_{i}+i+1} \int \omega_{1} \ldots \omega_{i-1}\left(\omega_{i} \wedge \omega_{i+1}\right) \omega_{i+2} \ldots \omega_{k}
\end{aligned}
$$

so that the graded submodule of the de Rham module on $\mathcal{P}_{x_{1}}^{x_{2}} M$ linearly generated by differential forms of the form $\int \omega_{1} \ldots \omega_{k}$ is closed under exterior differentiation, constituting a subcomplex of the de Rham complex $\mathcal{A}\left(\mathcal{P}_{x_{1}}^{x_{2}} M\right)$.

Definition 5.6. The subcomplex in the above theorem is called the bar complex associated with the de Rham complex $\mathcal{A}(M)$.

\section{REFERENCES}

[1] K.-T. Chen, Integration of paths - a faithful representation of paths by noncommutative formal power series, Trans. Amer. Math. Soc. 89 (1958), 395-407.

[2] K.-T. Chen, Iterated integrals of differential forms and loop space homology, Ann. Math. 97 (1973), 217-246.

[3] K.-T. Chen, Iterated path integrals, Bull. Amer. Math. Soc. 83 (1977), 831-879.

[4] V.G. Drinfel'd, On quasitriangular Hopf algebras and a group closely connected with Gal ( $\overline{\mathbf{Q}} / \mathbf{Q})$, Leningr. Math. J. 2 (1991), 829-860.

[5] B. Harris, Iterated Integrals and Cycles on Algebraic Manifolds, Nankai Tracts in Mathematics 7, World Scientific, Singapore, 2004.

[6] P. Iglesias-Zemmour, Diffeology, American Mathematical Society, Providence, Rhode Island, 2013.

[7] A. Kock, Synthetic Differential Geometry, 2nd ed., Cambridge University Press, Cambridge, 2006.

[8] T. Kohno, Vassiliev invariants of braids and iterated integrals, Adv. Stud. Pure Math. 27 (2000), 157-168.

[9] T. Kohno, Geometry of Chen's Iterated Integrals (in Japanese), Springer Japan, Tokyo, 2009.

[10] M. Konstevich, Vassiliev's knot invariants, Adv. Soviet Math. 16 (1993), 137-150.

[11] R. Lavendhomme, Basic Concepts of Synthetic Differential Geometry, Kluwer Academic Publishers, Dordrecht, 1996. 
[12] H. Nishimura, Axiomatic differential geometry I-1 - towards model categories of differential geometry, Math. Appl. 1 (2012), 171-182.

[13] H. Nishimura, Axiomatic differential geometry II-1 - vector fields, Math. Appl. 1 (2012), 183-195.

[14] H. Nishimura, Axiomatic differential geometry II-2 - differential forms, Math. Appl. 2 (2013), 43-60.

[15] A. Stacey, Comparative smootheology, Theory Appl. Categ. 25 (2011), 64-117.

[16] T. Terasoma, Mixed Tate motives and multiple zeta values, Invent. Math. 149 (2002), 339369.

Hirokazu Nishimura, Institute of Mathematics, University of Tsukuba, Tsukuba, Ibaraki, 3058571, Japan

e-mail: logic@math.tsukuba.ac.jp 
